\title{
4D Analysis of the Microstructural Evolution of Si-based Electrodes during Lithiation: Time-lapse X-ray Imaging and Digital Volume Correlation
}

\author{
J. M. Paz-Garcia, ${ }^{\mathrm{a}, *}$, O. O. Taiwo ${ }^{\mathrm{b}}$, E. Tudisco ${ }^{\mathrm{a}}$, D. P. Finegan ${ }^{\mathrm{b}}$, P. R. Shearing ${ }^{\mathrm{b}}$, D. J. L. Brett ${ }^{\mathrm{b}}$, S. A. Hall ${ }^{\mathrm{a}}$ \\ ${ }^{a}$ Division of Solid Mechanics, Lund University. Lund, Sweden \\ ${ }^{b}$ Electrochemical Innovation Lab, Department of Chemical Engineering, University College London. Torrington Place, London, UK
}

\begin{abstract}
Silicon is a promising candidate to substitute or complement graphite as anode material in Li-ion batteries due, mainly, to its high energy density. However, the lithiation/delithiation processes of silicon particles are inherently related to drastic volume changes which, within a battery's physically constrained case, can induce significant deformation of the fundamental components of the battery that can eventually cause it to fail.

In this work, we use non-destructive time-lapse X-ray imaging techniques to study the coupled electrochemomechanical phenomena in Li-ion batteries. We present X-ray computed tomography data acquired at different times during the first lithiation of custom-built silicon-lithium battery cells. Microstructural volume changes have been quantified using full 3D strain field measurements from digital volume correlation analysis. Furthermore, the extent of lithiation of silicon particles has been quantified in 3D from the grey-scale of the tomography images. Correlation of the volume expansion and grey-scale changes over the silicon-based electrode volume indicates that the process of lithiation is kinetically affected by the reaction at the $\mathrm{Si} / \mathrm{Li}_{x} \mathrm{Si}$ interface.
\end{abstract}

Keywords: Lithium-ion battery, Silicon electrode, X-ray computed tomography, Digital volume correlation

\section{Introduction}

Lithium-ion batteries (LIBs) are characterized by high energy density, low self-discharge, no memory effect, high efficiency and long cycle life [1]. These properties have made LIBs the preferable power source for the consumer electronics market [2], which drives forward the LIB technology together with the sectors of transportation and grid energy storage [3]. The development of LIB technology is expected to have an important effect on the popularization of environmentally friendly energy systems such as solar cells, wind power generation and electric vehicles [4]. There is, thus, a growing interest in the development of a new generation of LIBs, which should match the needs of high capacity, stable cyclability and safety of those technologies. In this context, intense research has been devoted to the investigation of materials to substitute existing electrodes, such as graphite for the negative electrode and lithiumcobalt oxide, lithium-manganese oxide and lithium iron phosphate for the positive electrode [5-7].

In the aforementioned traditional electrode materials, the lithiation process takes place by means of an intercalation mechanism. That is, ions occupy interstitial sites within a relatively rigid structures. For example, the graphene layers in the graphite electrode. In a charged battery with a graphite negative electrode, the lithium ions occupy interstitial spaces up to a stoichiometric composition of $\mathrm{LiC}_{6}$, presenting a theoretical capacity of $372 \mathrm{mAh} \mathrm{g}^{-1}[8,9]$.

Lithium-alloying materials, such as silicon, tin or aluminium have been proposed as candidates to substitute or complement the graphite electrode, due to their significantly higher gravimetric capacities. Among these alloying anode materials, silicon ( $\mathrm{Si}$ ) is one of the most promising due to its exceptionally high capacity and its abundance in the earth's crust [6]. The theoretical fully-lithiated phase, $\mathrm{Li}_{4.4} \mathrm{Si}$, has a capacity of approximately $4200 \mathrm{mAh} \mathrm{g}^{-1}$. In practice, it has been observed that the highest lithiated phase of $\mathrm{Si}$ at room temperature corresponds to $\mathrm{Li}_{3.75} \mathrm{Si}_{\text {, }}$

\footnotetext{
${ }^{*}$ Corresponding author

Email address: juanma.paz@me.com (J. M. Paz-Garcia)
} 
Table 1: Comparison of anode materials for LIBs [4, 8]

\begin{tabular}{llll}
\hline Materials & $\mathrm{Li}$ & $\mathrm{C}$ & $\mathrm{Si}$ \\
\hline Density $\left(\mathrm{g} \mathrm{cm}^{-3}\right)$ & 0.53 & 2.25 & 2.33 \\
Lithiated phase & $\mathrm{Li}$ & $\mathrm{LiC}_{6}$ & $\mathrm{Li}_{3.75} \mathrm{Si}$ \\
Gravimetric capacity $\left(\mathrm{mAh} \mathrm{g}^{-1}\right)$ & 3862 & 372 & 3579 \\
Volumetric capacity $\left(\mathrm{mAh} \mathrm{cm}^{-3}\right)$ & 1980 & 840 & 2440 \\
Volume change $(\%)$ & 100 & 12 & $>300$ \\
Potential vs. Li $(\approx \mathrm{V})$ & 0 & 0.05 & 0.15 \\
\hline
\end{tabular}

with a specific capacity of approximately $3579 \mathrm{mAh} \mathrm{g}^{-1}$, with a number of intermediate amorphous phases with stoichiometry $\mathrm{Li}_{x} \mathrm{Si}$ (with $0 \leq x \leq 3.75$ ) [10-12]. Table 1 compiles some properties of the anode materials of interest in this study.

Alloying electrodes experience extreme volume changes during lithiation, over $300 \%$ for Si [13]. These huge volume changes produce mechanical deformations and damage to the fundamental components of a battery, causing rapid and severe capacity loss leading to battery failure. Irreversible capacity loss, particularly during the first lithiation step, has been reported to be caused mainly by: (1) the loss of active material, as the active particles crack and pulverize leading to the disconnection of the alloy particles from the current collector, binder and the conductive carbon; (2) the continuous formation of solid-electrolyte interphase (SEI) and volume changes that produce the detachment of the SEI, which reforms in every cycle causing irreversible loss [8].

Different options have been proposed to mitigate the negative effect of these volume changes. One of the most promising solutions is the use of silicon nanoparticles, nanotubes or nanowires, where the reduced particle size seems to produce more stable lithation/delithiation cycles. However, large specific surface areas lead to high levels of SEI formation. In this context, Liu et al. [14] proposed a yolk-shell structure to mitigate volume changes; the structure consists of a carbon-based shell around each nano-sized Si particle aimed at forming a stable mechanical framework upon Si volume expansion. The well-defined void space allows the Si particles to expand freely without breaking the outer carbon shell, therefore stabilizing the solid-electrolyte interphase on the shell surface [15].

The mechanical consequences of the electrochemical processes that take place in battery electrodes play a crucial role in determining battery performance and lifetime [16]. A better understanding of these processes is essential to achieve new generations of LIBs based on alloying electrodes. In this study, we use 4D X-ray computed tomography (XCT) imaging techniques to visualize the evolution of Si-based electrodes during the first lithiation step.

XCT enables high-resolution, non-invasive, non-destructive 3D imaging for the qualitative and quantitative inspection of material structure and properties. XCT has been demonstrated for the visualization of the battery components' micro-structural evolution at multiple length scales and as an effective tool for diagnosing battery failure mechanisms post-mortem $[17,18]$. XCT has previously been used to explore the micro-structural properties of electrode materials in LIBs [19-22]. Furthermore, consecutive 3D images during the operation allows the 4D (3D plus time) analysis, including the possibility to carry out tests under in-situ or in-operando conditions (XCT during, for example, electrochemical tests). For cells similar to those studied in this work, Gonzalez et al. $[5,23]$ investigated the evolution of Si electrodes during the first lithiation and Sn electrodes during the first lithiation/delithiation cycle.

In this study, we use Digital Volume Correlation (DVC) (e.g., $[24,25])$ to quantify the mechanical deformation of the fundamental cell components (electrodes and separator) during the lithiation process. DVC has been demonstrated as a useful tool for the diagnosis of electrochemical devices [18, 26], but has not been used yet (to the authors knowledge) for the study of lithium-alloying electrodes.

\section{Experimental and Methods}

\subsection{Electrode preparation and cell assembly}

Lithium-silicon half cells (Fig. 1) were assembled within thin-walled X-ray transparent PFA Swagelok cells [21]. Electrode slurry was prepared by mixing micron-sized Si powder (300 mesh, $99 \%$ purity), conductive graphite and polyvinylidene flouride (PVDF) binder in the respective weight ratios 80:10:10 with n-methyl-2-pyrrolidone (NMP) using a mortar and pestle. All chemicals used in the slurry preparation were acquired from Pi-Kem Ltd., UK. The 
resulting slurry was then dip-coated onto one tip of a steel current collecting pin (ca. $3.2 \mathrm{~mm}$ diameter), and the coated pins were placed in a vacuum oven to dry overnight at $80^{\circ} \mathrm{C}$. The active Si loading was estimated by weighing the current collecting pins before and after dip coating. Half cells were assembled in an argon-filled glove-box $\left(\mathrm{O}_{2}<2\right.$ ppm, $\mathrm{H}_{2} \mathrm{O}<0.5 \mathrm{ppm}$ ) with metallic lithium foil counter electrode and a borosilicate flass-fibre separator (Whatmann GF-D grade, GE). The electrolyte used consists of $1 \mathrm{M} \mathrm{LiPF}_{6}$ dissolved in a mixture of ethylene carbonate (EC) and ethyl methyl carbonate (EMC) (in the ratio 3:7 by weight). The cells were assembled at the Electrochemical Innovation Lab of the Department of Chemical Engineering, University College London.

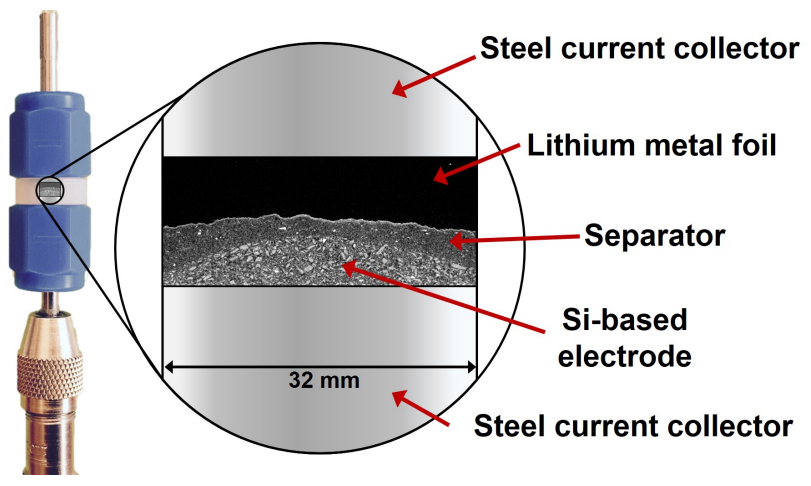

Figure 1: Scheme of the manufactured Swagelok cells.

The Si electrodes were tested against lithium metal. Accordingly, in these half cells, the lithium metal acts as the negative electrode and the silicon as the positive electrode [27].

\subsection{Electrochemical and XCT tests}

An assembled cell with theoretical capacity of $c a$. $7.45 \mathrm{mAh}$ (calculated based on the Si content and assuming lithiation to $\mathrm{Li}_{3.75} \mathrm{Si}$ ), was galvanostatically discharged using a potentiostat (Ivium Compactstat, Ivium Technologies). XCT was performed using Xradia Versa MicroXCT-520 laboratory tomograph at the 4D-Imaging lab (Division of Solid Mechanics, Lund University). The cell was partially discharged at successive intervals in 10 consecutive steps at $25 \mu \mathrm{A}$. The first step lasted $10 \mathrm{~h}$ and the electrode was lithiated $3.36 \%$. Consecutive steps had $20 \mathrm{~h}$ duration each, and the electrode was lithiated $\approx 6.72 \%$ in each step. Further lithiation of the cell after the 10th step was not possible, probably due to breakdown of the separator, as discussed in the results sections. Figure 2 shows the cell potential readings for the 10 steps of partial lithiation of the $\mathrm{Si}$ electrode.

A total of 11 XCT scans were carried out (one for the initial state and after each of the 10 partial discharge steps). For the scans, the source and the detector were set at $15 \mathrm{~mm}$ distance (in front of and behind to, respectively) the center of the sample, achieving a pixel size of $1.7 \mu \mathrm{m}$ using a $4 \times$ objective. Scans were carried out with a source tube

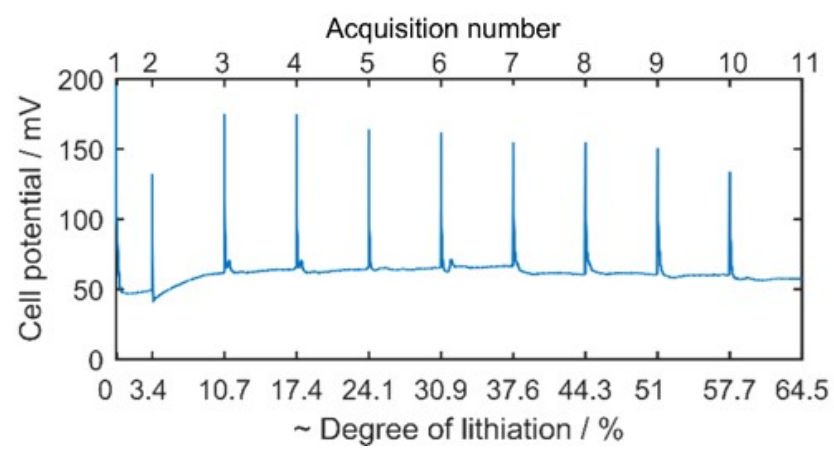

Figure 2: Partial discharge steps during the galvanostatic lithiation of the Si electrode. Current: $25 \mu \mathrm{A}$. Duration of the step: $20 \mathrm{~h}$ per step (10 h in the case of the first step). After each partial discharge step, XCT scans were made. 
voltage of $45 \mathrm{kV}$ with exposure time of $30 \mathrm{~s}$ per projection, acquiring 2001 projections per scan. The reconstructed tomograms consisted of 16 bit grey-scale image stacks of $2000 \times 2000$ pixels per image.

\subsection{DVC analysis}

DVC was used to quantify the micro-structural evolution of the cells between the successive discharge steps. The DVC technique consists of the comparison of two volumes (3D image acquired via XCT) to identify the displacement field that maps the deformed volume on to the original volume. The DVC provides full 3D vector displacement fields, which are then interpreted following continuum mechanics to provide 3D tensor-strain maps.

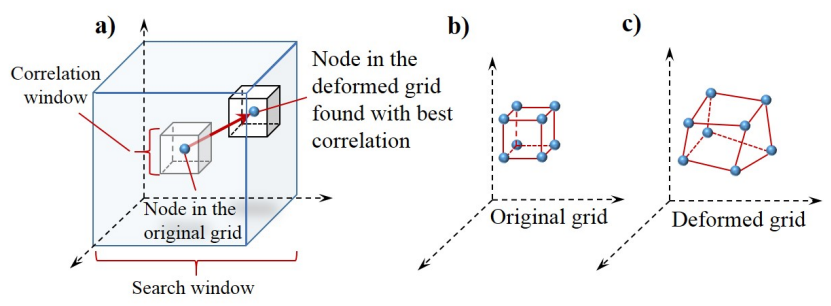

Figure 3: Digital volume correlation. (a) Schematic representation of the displacement vector for a sample node. (b) Cubic subvolume limited by 8 neighbour nodes in the original regular grid. (c) Deformed subvolume in the displaced grid.

The DVC analysis was carried out using the DVC software Tomowarp2 [28], Python-based code developed between the Universities of Grenoble and Lund. This DVC algorithm includes sub-pixel resolution of the displacements and consists of the following steps. First, a 3D grid of nodes is defined in the reference volume. Around each node, a correlation window (subvolume centred on the node in the original volume) is defined. The corresponding correlation window is sought within the deformed volume, over a 3D region denoted as search window (see Fig. 2.3.a). During the search, the subvolumes are compared by a correlation coefficient calculated at each position.

One of the challenges addressed in this research is to investigate whether it is possible to use DVC to follow the volume changes in the $\mathrm{Si}$ electrode during the lithiation process, which is undergoing chemical transformation, with associated attenuation changes, in addition to displacements. In this context, it is assumed that the lithiation steps applied to the cells are small enough to allow a proper correlation between two consecutive images in the sequence of acquisitions. Consequently, it should be possible to carry out a study of the incremental deformation of the battery during the lithiation process.

\section{Results and Discussion}

\subsection{XCT results}

Figures 4 and 5 show, respectively, 6 vertical and 3 horizontal cross-section images from the 11 acquired image volumes. In both figures the grey-scale range has been adjusted to the threshold limit between 13750 and 18250 (16 bit). This threshold leaves the lithium metal near the lower saturation limit. Materials with higher density, such as Si particles and glass fibre separator, are marked by high grey-scale values. A small number of particles are observed with X-ray attenuation much higher than Si (bright saturated in the images). These particles were assumed to be impurities in the original Si powder.

The lithiation process is clearly accompanied by a significant volume expansion of the Si-based electrode. The volume of the electrode at $64.5 \%$ lithiation is nearly three times that of the initial state. The separators mechanical stability contributes immensely to the safety and performance of LIB cells. The huge volume changes induced the rupture of the separator in the middle part with through-going cracks. It was impossible to further lithiate the electrode over the value of $64.5 \%$ due, presumably, to a short-circuit between the two electrodes.

In the cell studied here, the Si electrode was tested against a lithium metal electrode, whose volume decreases as the lithium oxidises into lithium ions. During the lithiation, the separator suffered a vertical displacement towards the lithium electrode, pushed by the growing Si-based electrode. The separator also suffered compression, indicating than the expansion of the the Si electrode exceeds the volume released at the lithium metal electrode. 

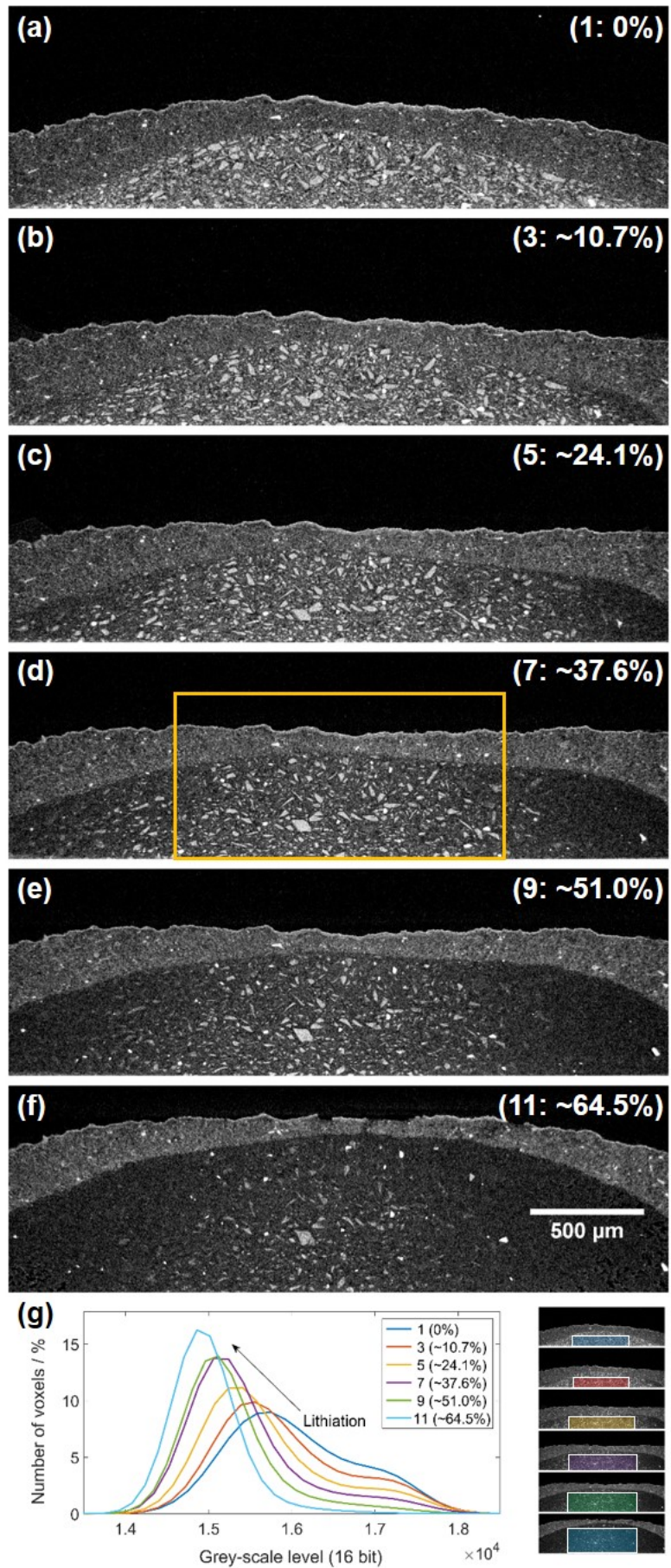

Figure 4: (a-f) Vertical cross sections of the cell at different lithiation degrees. 6 images (16 bits images and grey-scale of bulk with threshold limits 13750 - 18250) are shown within a total of 11 XCT acquisitions. Inset brackets indicate the acquisition number and the degree of lithiation of the electrode. Yellow rectangle in (d) indicates the limits of the subvolume analysed in Fig. 7 (g) Colour depth histogram of a subvolume in the Si-based electrode, capturing the change in attenuation at different states of electrode lithiation. Coloured boxes, to the right, indicate the subvolumes over the expanding electrode where the colour depth histograms were computed. 

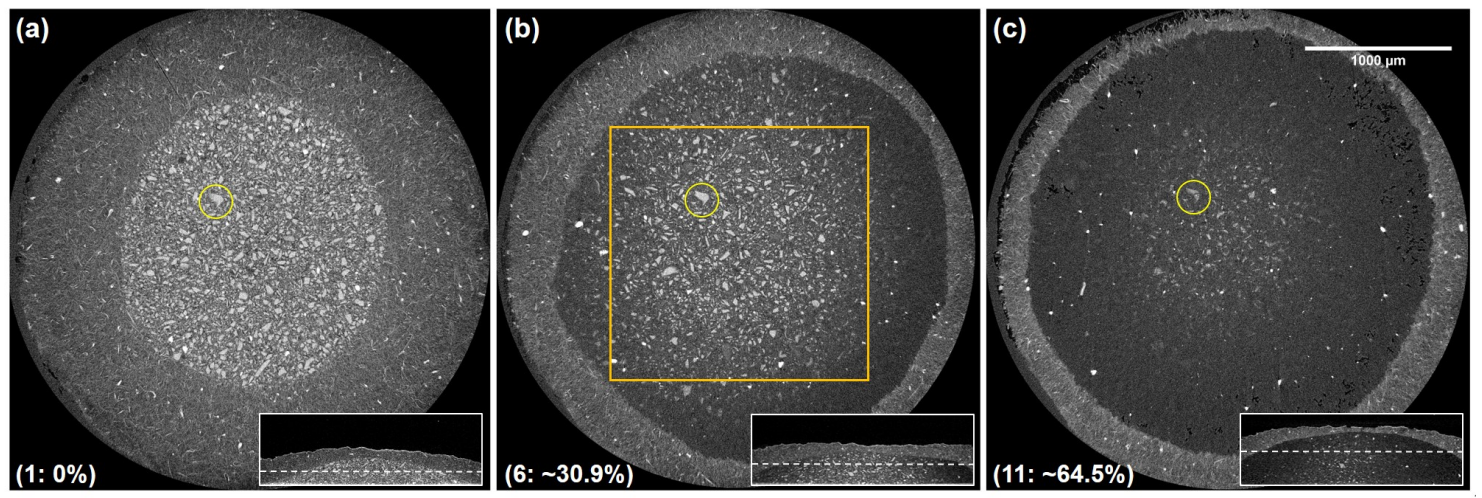

Figure 5: Horizontal cross-section images of the cell at different degrees of electrode lithiation. 3 images within a total of 11 XCT acquisitions are shown. 16 bit images and grey-scale with threshold limits 13750 and 18250. Inset brackets indicate the acquisition number and the corresponding degree of lithiation of the electrode. The vertical position of the slice is indicated in an inset image. A selected particle is indicated with a circle. Yellow rectangle in (b) indicates the limits of the subvolume analysed in Fig. 7

Moreover, in the tested cell, the separator exhibited uneven initial thickness indicating certain level of compression in the central part of the cell. This initial compression, resulting from the manufacturing process, is related to a decrease of the separator's porosity in that central region. As a consequence of the the local decrease of the lithium diffusivity in that central region, lithium ions seems to go across the separator preferentially through the outer region. The lithiation front (i.e., the front of decrease of the attenuation in the images) is observed to progress in radial direction from the upper-outer towards the inner-central part of the electrode.

Despite the narrow grey-scale range needed to appreciate contrast between the phases, the change of the attenuation in the Si electrode during the lithiation process is clearly observed. As the lithiation takes place, the Si particles react with the lithium ions to form lithiated-Si phases $\left(\mathrm{Li}_{x} \mathrm{Si}\right)$ which have lower X-ray attenuation and, therefore, appear darker. The impurities did not experience either volume nor attenuation changes during the process. Figure 4.g shows the grey-scale histogram for subvolumes corresponding to the same region in the Si electrode (i.e. excluding the separator and the lithium foil region) taking into account the dilation of the electrode. The fresh cell presents a histogram with two overlapped peaks centred on 15800 and 17000 . The lower grey-scale peak is related to the electrode matrix (mainly consisting of conductive carbon, binder and pores filled with electrolyte) and the outer boundary of the silicon particles. The peak in the histogram centred on a grey-scale of at 17000 is related to the core of the silicon particles (non-lithiated $\mathrm{Si}$ ). The histogram evolution during the lithiation process shows a clear decrease of the attenuation, towards a single peak centred on approximately 14900 , which is inferred to correspond to the lithiated-Si phase.

As the lithiated-Si phase has an X-ray attenuation factor very similar to the electrode's conductive matrix, it is easier to distinguish the shrinking $\mathrm{Si}$ core of the particles than the expansion of the outer lithiated-Si region. Figure 6 shows a selected particle at 3 different states of electrode lithiation. The chosen particle, which is also indicated in Fig. 5, does not experience significant attenuation and volume changes until the fifth acquisition. It must be noted that the degree of lithiation of the particle is not the same as the lithiation degree of the bulk electrode, since the lithiation does not take place homogeneously within the cell.

Fig. 6 shows the evolution of the particle through the lithiation and, in particular, shows detail in the reducing size of the unlithiated core. This figure is constructed as follows: first, the selected particle was identified and isolated in the chosen acquisitions (7,9 and 11) and subvolumes around the particle were extracted. Subsequently, the subvolume was thresholded to make voxels with a grey-scale value less than 16250 transparent in the final volume renderings shown in the figure. The resultant visible volume of the particle (in Fig. 6.a-c) which corresponds to the unlithiated Si in the particle was approximately 18000, 15000 and 9000 voxels respectively (the latter being the combined volume of the two pieces of the divided particle in Fig. 5.c).

Figure 6.d shows the same particle shown in Fig. 6.c, but constructed using a dilated mask, to cover not only the segmentated particle but also the surrounding of that particle. The surrounding region is faded (presented with certain level of transparency) in order to distinguish the inner and the outer part. The fade function is included in the inset 
(a)

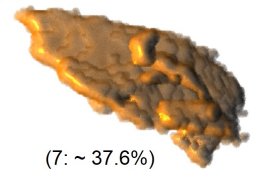

(b)

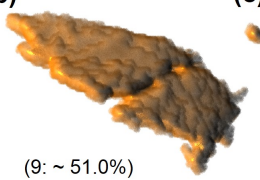

(c)

(d)

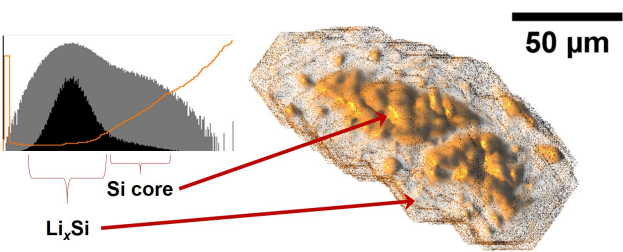

Figure 6: (a-c) A selected particle located in the central part of the Si electrode at three different states of electrode lithiation. The particle represents only the non-lithiated $\mathrm{Si}$ core, which shrinks whilst lithiation takes place. Inset brackets indicate the acquisition number and the corresponding degree of lithiation of the Si electrode. (d) The Si core is identified with a threshold over 16250 (16 bits). The wider mask shows that around the Si core there is a region of lithiated-Si with lower X-ray attenuation.

histogram. Fig. 6.d shows that there is a clear difference in the attenuation level of the particle between the inner unlithiated core and the outer lithiated region, and therefore, it is possible to make a segmentation between the Si core and the lithiated-Si phase.

\subsection{DVC results}

In this section, results from the DVC analysis of the sequence of acquisitions are presented. The results were obtained using a 3D grid with 25 voxels node spacing and a DVC window of \pm 25 voxels in $\mathrm{X}, \mathrm{Y}$ and Z. Correlation windows were, therefore, cubic subvolumes of 51 voxels side $(\approx 86 \mu \mathrm{m}$ side). The DVC computation was carried out over a subvolume located in the central part of cell with size $1000 \times 1000 \times 500$ voxels $\left(\approx 0.845 \mathrm{~mm}^{3}\right)$, including the Si-based electrode, the separator and a fraction of the lithium foil (see Fig. 7.d).
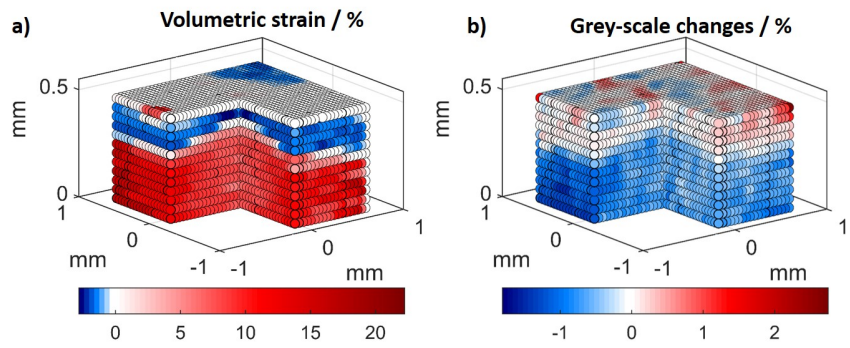

c)

d)
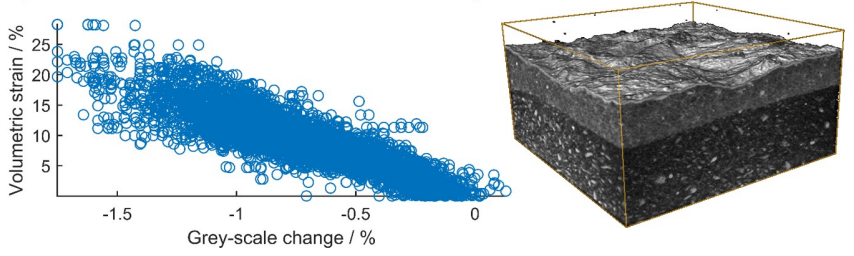

Figure 7: DVC results between the acquisitions $6(\approx 30.9 \%)$ and $7(\approx 37.6 \%)$. (a) 3D volume strain field. (b) local grey-scale changes at the same positions as the local DVC subvolumes. Axis in $\mathrm{mm}$. $\mathrm{X}$ and $\mathrm{Y}$ axis referred to the centre of the cylindrical cell. $\mathrm{Z}$ axis referred to the current collector below the Si-based electrode. (c) Local relation between the volumetric strain and the grey-scale changes for the electrode region. d) 3D rendering of the subvolume used for the DVC analysis. The limits of the subvolume are indicated in the figures 4.d and 5.b

Figure 7.a shows the results obtained using the DVC algorithm between two consecutive acquisitions of the cell, namely acquisitions 6 and 7 , corresponding to global lithiation degree of $\approx 30.9 \%$ and $\approx 37.6 \%$, respectively. In Fig. 
7.a, the 3D field values are shown using a blue-white-red chromatic scale, where red (positive) means expansion and blue (negative) means compression. Fig. 7.b shows the corresponding results for the local grey-scale changes, where red (positive) means an increase, and blue (negative) means a decrease of the grey-scale level. The local change of the grey-scale values were computed for the same subvolumes where the volumetric strain was calculated from the DVC.

In the upper regions of Fig. 7.a-b, i.e., in the separator, mainly volume reduction is observed and a slight increase in grey-level, which are both consistent with compression and densification of the porous separator. The lower regions of Figure 7.a-b correspond to the Si-based electrode. The DVC (Fig. 7.a) indicates positive volumetric strain (expansion) during the lithiation in this region. Meanwhile, Fig. 7.b shows that there is a clear decrease in the grey-level (decrease in X-ray attenuation) in the electrode. This is consitent with the global the results presented in Fig. 4.g and in correspondence with the Si lithiation producing a decrease in the particle density.

It is possible to study the relation between the volumetric strain and the grey-scale changes in a local scale, i.e. at the different positions of the electrode where the degree of lithiation may be different due to the in-homogeneous lithiation. Fig. 7.c shows an example of the local relation between the volumetric-strain and the grey-scale changes in the region of interest selected within the Si electrode, for the same values presented in Fig. 7.a. At a given step, the local grey-scale change and volumetric strain are linearly related.

Based on the different mechanical behaviour of the Si-based electrode and the separator, it is possible to do a segmentation between the two in the volumetric strain map. In particular, we consider that the region of the subvolumes exhibiting positive volumetric strain (expansion) belongs to the electrode. Figure 8 shows the results of the DVC for each pair of consecutive acquisitions, showing only the section experiencing volume expansion. The figure shows that the local volumetric strain in the Si electrode increases as the lithiation progresses. The global incremental increase in lithiation over each of these steps is the same, but it can be seen that the degree of volumetric strain calculated over each increment increases as the total degree of lithiation increases.

Figure 8.k presents the computed accumulated values for the volume expansion within the region of electrode studied as a function of the average degree of lithiation. A polynomial fitting curve is included, indicating that the computed volumetric strain relates to the bulk electrode lithiation degree in a quadratic manner. The volume expansion increases with lithiation, meaning that the Si electrode exhibits more volume expansion at higher degrees of lithiation. At $\approx 64.5 \%$ electrode lithiation, the volume increment is calculated to be ca. $155 \%$. Extrapolating the second degree curve to 100 lithiation gives a predicted volume expansion of 350, which is consistent with the volume expansion reported in the literature $[5,6]$ for the full lithiation of Si particles.

Figure 8.k also shows the global grey-scale changes in the electrode, referred to the original average grey-scale value. Local grey-scale changes were computed in two different ways: using each of the corresponding subvolume from the 3D strain analysis, and then averaged for the entire electrode at each lithiation step, and averaging from the histogram values presented in Fig. 4.g. Local grey-scale results show strong agreement between the two methods methods. The grey-scale change follows a linear trend with respect to the global lithiation degree.

The lithiation process of silicon particles can be considered as the combination of different processes in series; namely: (1) the redox reaction at the electrolyte/silicon interface, (2) the diffusion of lithium through the lithiated phase, and (3) the chemical reaction at the $\mathrm{Si} / \mathrm{Li}_{x} \mathrm{Si}$ interface [11]. As the lithiation process is carried out galvanostatically, the overall rate of the reduction reaction of lithium on the surface of the particles along the electrode is constant. However, due to the observed in-homogeneous transport of lithium ions through the separator, the rate of lithiation of the particles depends on the position in the Si-based electrode. The particles in the outer region lithiate faster than the particles in the centre of the cell.

XCT results showed that it is possible to distinguish between the non-lithiated core and the lithiated shell with diffusing lithium atoms. DVC results, in turn, show that the volume changes of the Si electrode increase with the lithiation degree in a quadratic manner, while the grey scale decreases linearly. These observations suggest that: (i) the change in local grey-scale value can be used as a proxy for the local lithiation degree; (ii) the lithiation process is affected by the available surface area at the $\mathrm{Si} / \mathrm{Li}_{x} \mathrm{Si}$ interface where the lithiation reaction (i.e., the breaking of $\mathrm{Si}-\mathrm{Si}$ bonds and formation of Li-Si bonds) takes place, since, if the lithiation rate is constant, the change in surface area will be quadratic. 


\section{Conclusions}

This work presents results for XCT observations of the 4D microstructural evolution of Si-based porous electrodes during the first lithiation process. The presented results demonstrate that it is possible to carry out the quantification of the micro-structural evolution of lithium battery components using XCT imaging in combination with DVC.

In Si-based porous electrodes, as the lithiation takes place, the X-ray attenuation decreases and the volume of the active particles expands. Both effects are efficiently tracked by XCT and DVC. In the cell tested here, the coupled electrochemical and mechanical phenomena causes the breakdown of the separator, and the eventual short-circuit of the cell. Furthermore, the non-homogeneous compression of the separator is related to preferential paths for the transport of lithium ions during the cell operation.

The quantifications of the volume and grey-scale changes from the images show that the volume changes of the $\mathrm{Si}$ electrode increase with the lithiation degree in a quadratic manner, while the grey scale (i.e., the X-ray attenuation) decreases linearly. These observations suggest that: (i) the change in local grey-scale value can be used as a proxy for the local lithiation degree; (ii) the lithiation process is affected by the available surface area at the $\mathrm{Si} / \mathrm{Li}{ }_{x} \mathrm{Si}$ interface where the lithiation reaction takes place. Furthermore, extrapolation of the quadratic trend in volume change to $100 \%$ lithiation indicates a maximum volume expansion of $\approx 350 \%$, which suggests that operating the cell below certain threshold limit of the lithiation degree may increase the durability and increase the specific capacity of the cells.

\section{Acknowledgements}

The authors acknowledge the financial support from the International Campus of Excellence (ICE) Andalucia Tech and the Global Challenge Network in Batteries and Electrochemical Energy Device of the Science and Technology Facilities Council, the Faculty of Engineering in Lund University, and the Crafoord Foundation (\#20140802). X-ray tomography was performed at the 4D-Imaging Lab (Division of Solid Mechanics, Lund University).

\section{References}

[1] D. Miranda, C. Costa, S. Lanceros-Mendez, Lithium ion rechargeable batteries: State of the art and future needs of microscopic theoretical models and simulations, Journal of Electroanalytical Chemistry (739) (2015) 97-110.

[2] B. Scrosati, J. Garche, Lithium batteries: Status, prospects and future, Journal of Power Sources (195) (2010) 2419-2430.

[3] B. Diouf, R. Pode, Potential of lithium-ion batteries in renewable energy, Renewable Energy 76 (2015) 375-380.

[4] K. Nishikawa, H. Munakata, K. Kanamura, In-situ observation of one silicon particle during the first charging, Journal of Power Sources 243 (2013) 630-634.

[5] J. Gonzalez, K. Sun, M. Huang, J. Lambros, S. Dillon, I. Chasiotis, Three dimensional studies of particle failure in silicon based composite electrodes for lithium ion batteries, Journal of Power Sources 269 (2014) 334-343.

[6] M. T. McDowell, S. W. Lee, W. D. Nix, Y. Cui, 25th anniversary article: Understanding the lithiation of silicon and other alloying anodes for lithium-ion batteries, Advanced Materials 25 (2013) 4966-4985.

[7] N. Nitta, F. Wu, J. T. Lee, G. Yushin, Li-ion battery materials: Present and future, Materials Today 18 (2015) $252-264$.

[8] W. Zhang, A review of the electrochemical performance of alloy anodes for lithium-ion batteries, Journal of Power Sources 196 (2011) 13-24.

[9] G. Pistoia, Lithium-Ion Batteries Advances and Applications, Elsevier, Amsterdam, 2014.

[10] V. A. Sethuraman, V. Srinivasan, J. Newman, Analysis of electrochemical lithiation and delithiation kinetics in silicon, Journal of the Electrochemical Society 160 (2013) A394-A403.

[11] M. Pharr, K. Zhao, X. Wang, Z. Suo, J. J. Vlassak, Kinetics of initial lithiation of crystalline silicon electrodes of lithium-ion batteries, Nano Letters 12 (2012) 5039-5047.

[12] K. Ogata, E. Salager, C. Kerr, A. Fraser, C. Ducati, A. Morris, S. H. C. Grey, Revealing lithiumsilicide phase transformations in nanostructured silicon-based lithium ion batteries via in-situ NMR spectroscopy, Nature Communications 5 (2014) 1-11.

[13] H. Yang, F. Fan, W. Liang, X. Guo, T. Zhu, S. Zhang, A chemo-mechanical model of lithiation in silicon, Journal of the Mechanics and Physics of Solids 70 (2014) 349-361.

[14] N. Liu, H. Wu, M. T. McDowell, Y. Yao, C. Wang, Y. Cui, A yolk-shell design for stabilized and scalable Li-ion battery alloy anodes, Nano Letters 12 (2012) 3315-3321.

[15] M. B. Pinson, M. Z. Bazant, Theory of SEI formation in rechargeable batteries: Capacity fade, accelerated aging and lifetime prediction, Journal of the Electrochemical Society 160 (2013) A243-A250.

[16] J. Cannarella, C. B. Arnold, Stress evolution and capacity fade in constrained lithium-ion pouch cells, Journal of Power Sources 245 (2014) $745-751$.

[17] P. Shearing, Y. Wu, S. J. Harris, N. Brandon, In situ X-ray spectroscopy and imaging of battery materials, The Electrochemical Sociecty Interface Fall (2011) 43-47.

[18] D. P. Finegan, E. Tudisco, M. Scheel, J. B. Robinson, O. O. Taiwo, D. S. Eastwood, P. D. Lee, M. D. Michiel, B. Bay, S. Hall, G. Hinds, D. J. L. Brett, P. R. Shearing, Quantifying bulk electrode strain and material displacement within lithium batteries via high-speed operando tomography and digital volume correlation, Advance Materials 0 (2015) 1500332(1-11). 
[19] J. McBreen, The application of synchrotron techniques to the study of lithium-ion batteries, Journal of Solid State Electrochemistry 13 (2009) $1051-1061$.

[20] P. Shearing, L. Howard, P. Jrgensen, N. Brandon, S. Harris, Characterization of the 3-dimensional microstructure of a graphite negative electrode from a li-ion battery, Elecctrochemical Communications 12 (2010) 374-377.

[21] M. Ebner, F. Marone, M. Stampanoni, V. Wood, Visualization and quantification of electrochemical and mechanical degradation in Li-ion batteries, Science 342 (2013) 716-720.

[22] D. P. Finegan, M. Scheel, J. B. Robinson, B. Tjaden, I. Hunt, T. J. Mason, J. Millichamp, M. D. Michiel, G. J. Offer, G. Hinds, D. J. Brett, P. R. Shearing, In-operando high-speed tomography of lithium-ion batteries during thermal runaway, Nature Communications 6:6924 (2015) $1-10$.

[23] J. Gonzalez, K. Sun, M. Huang, S. Dillon, I. Chasiotis, J. Lambros, X-ray microtomography characterization of Sn particle evolution during lithiation/delithiation in lithium ion batteries, Journal of Power Sources 285 (2015) $205-209$

[24] S. A. Hall, J. Desrues, G. Viggiani, P. Bsuelle, E. Ando, Experimental characterisation of (localised) deformation phenomena in granular geomaterials from sample down to inter- and intra-grain scales, Procedia IUTAM 4 (2012) 541-65.

[25] M. Sjdahl, C. R. Siviour, F. Forsberg, Digital volume correlation applied to compaction of granular materials, Procedia IUTAM 4 (2012) 179 $-195$.

[26] D. S. Eastwood, V. Yufit, J. Gelb, A. Gu, R. S. Bradley, S. J. Harris, D. J. L. Brett, N. P. Brandon, P. D. Lee, P. J. Withers, P. R. Shearing, Lithiation-induced dilation mapping in a lithium-ion battery electrode by $3 \mathrm{D}$ X-ray microscopy and digital volume correlation, Advance Energy Materials 4 (2014) 1300506(1-7).

[27] A. Ferrese, P. Albertus, J. Christensen, J. Newman, Lithium redistribution in lithium-metal batteries, Journal of the Electrochemical Society 159 (10) (2012) A1615-A1623.

[28] E. Tudisco, S. Hall, E. Charalampidou, N. Kardjilov, A. Hilger, H. Sone, Full-field measurements of strain localisation in sandstone by neutron tomography and 3D-volumetric digital image correlation, Physics Procedia 69 (2015) 509-515. 
(a)

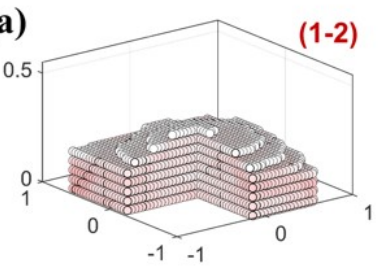

(c)

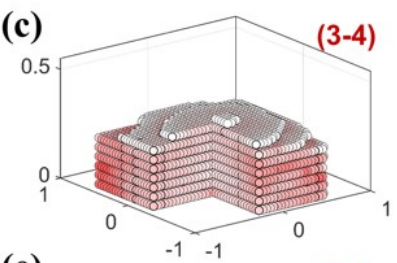

(e)

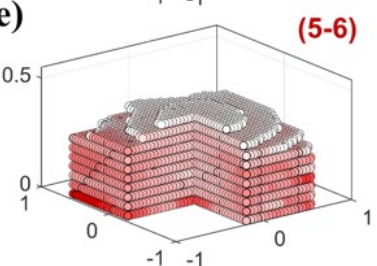

(g)

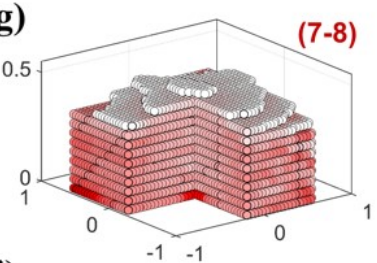

(i)

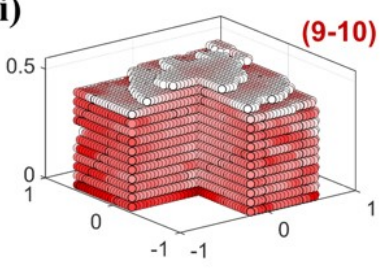

(b)

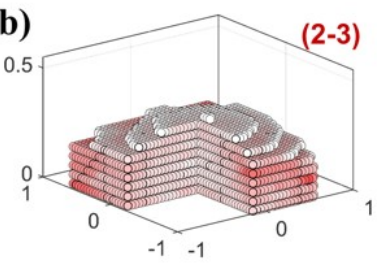

(d)

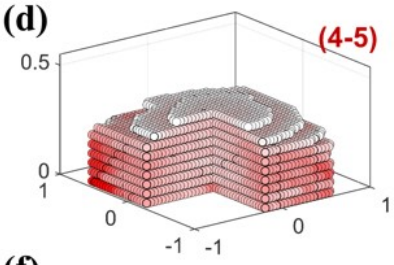

(f)

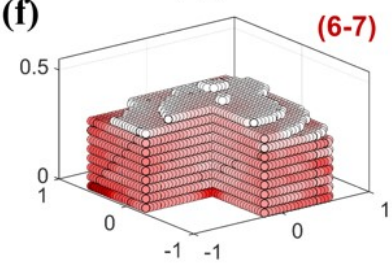

(f)

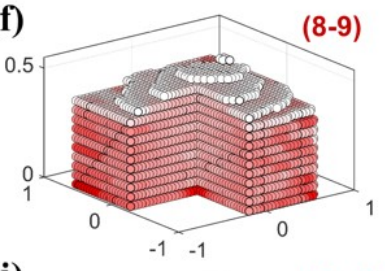

(j)

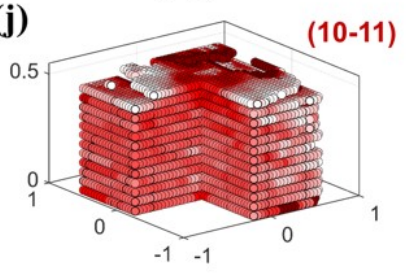

(k)
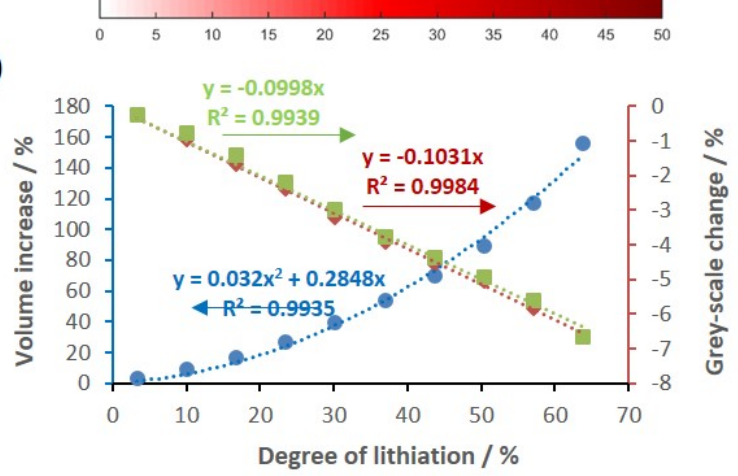

Figure 8: Volumetric strain computed via DVC, corresponding to each pair of consecutive acquisitions of the tested cell (indicated in brackets). Only the region exhibiting volume expansion (the Si electrode) is shown here. The color bar is common for all images, units in percentage of volume change. Axis in $\mathrm{mm}$. $\mathrm{X}$ and $\mathrm{Y}$ axis referred to the centre of the cylindrical cell. $\mathrm{Z}$ axis referred to the current collector below the Si-based electrode. (k) Volume increment of the electrode (blue-circles, left axis) and grey-scale changes (right axis) with referred to the original nonlithiated electrode. Grey-scale changes computed through data from the histogram in Fig. 4.g (green-squares) and averaging the local grey-scale changes computed via the DVC code (red-triangles) 